\title{
Echinacea-Präparate zurückhaltend einsetzen
}

\section{Echinacea-Extrakte werden bei uns gerne zur Prophylaxe von Erkältungskrankheiten eingenommen. Doch können diese Mittel teilweise lebensbedrohliche Allergien auslösen. Da ein endgültiger \\ Wirksamkeitsnachweis bisher fehlt, sollte speziell Allergikern von einer Einnahme abgeraten werden.}

Mit Beginn der Erkältungssaison haben bei uns auch wieder Präparate mit Extrakten aus Echinacea-Spezies, insbesondere Echinacea purpurea, dem Purpurfarbenen Sonnenhut, Hochkonjunktur. So wurde 1996 in Deutschland mit mehr als 500 Echinacea-haltigen Produkten ein Umsatz von über 45 Millionen DM erzielt. Hauptindikationsgebiet: die Prophylaxe von Erkältungskrankheiten.

„Unspezifische Immunstimulation“" in vitro und in vivo nachgewiesen Eine Zulassung erhielten diese Extrakte u.a. aufgrund eines in vitro und in vivo nachgewiesenen „unspezifischen immunstimulierenden" Effekts. Nachdem die Diskussion um eine mögliche krebsauslösende Wirkung durch diese Extrakte abgeklungen ist, wird immer wieder über teilweise lebensbedrohliche allergische Reaktionen nach Einnahme von Echinacea-Präparaten berichtet. Auch die Arzneimittelkommission der Deutschen Ärzteschaft hat sich bereits 1996 wegen der bekanntgewordenen Allergien gegen die Anwendung dieses Extrakts ausgesprochen (Höffler D. Arzn Verord Prax 1996; 3: 7).

Dies wiegt um so schwerer, als daß es zwar viele Studien mit einem positiven Wirksamkeitsnachweis für Echinacea gibt, ein wirklich wissenschaftlich anerkannter Beweis für eine klinisch relevante Prävention bei Erkältungskrankheiten aber bisher fehlt. Dies dokumentiert auch eine aktuelle randomisierte, plazebokontrollierte Studie mit 108 Probanden, bei der sich statisch kein Unterschied in der vorbeugenden
Wirkung zwischen Plazebo und Echinacea feststellen ließ (Grimm W, et al. Am J Med 1999; 106: 138).

Eine sorgfältige Nutzen-Risiko-AbPräparaten ist deshalb insbesondere bei Allergikern unbedingt anzuraten. $b k$

Der Arzneimittelbrief 1999; 33: 31-2. wägung vor Einnahme von Echinacea-

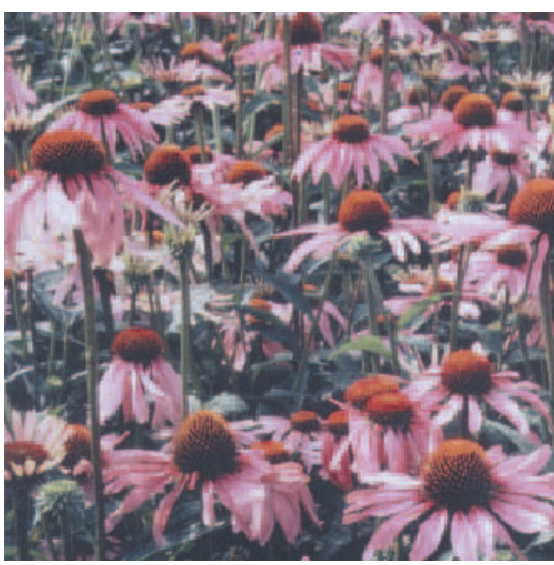

Echinacea purpurea, der Purpurfarbene Sonnenhut. Die Pflanze stammt aus Nordamerika, wo sie von den Indianern als Heilmittel zur Behandlung von Erkältungskrankheiten verwendet wurde. Heute genießt der Extrakt nur noch in Europa Popularität, besonders in Deutschland. Wie Kamille und Salbei, deren allergenes Potential seit längerem bekannt ist, gehören die Echinacea-Arten zur Familie der Korbblütler.

\section{Unveröffentlichte Studie sorgt für Aufsehen: Erhöhtes Atopierisiko durch Pertussisvakzine?}

Gegen Pertussis immunisierte Kinder haben ein um $75 \%$ höheres Risiko, später an Heuschnupfen, Asthma oder einem atopischen Ekzem zu erkranken. Diese Warnung ergibt sich aus den Ergebnissen einer bisher allerdings noch nicht publizierten Studie, durchgeführt am Cambridger Addenbrooke's Hospital und an der University of Wales, die jetzt bei einem Treffen des Royal College of Paediatrics and Child Health in New York präsentiert wurde.

Einbezogen in die Studie waren insgesamt 1934 zwischen 1975 und 1984 geborene Kinder, die als Patienten in einer Allgemeinpraxis in Oxfordshire registriert waren. Man analysierte ihre Daten in Hinsicht auf Impfungen, Infektionen und deren Therapie sowie auf das Vorliegen von Allergien in Form von Asthma, Rhinitis oder Ekzemen. Dabei stellte sich u.a. heraus, daß eine Impfung mit einer ganzzelligen Pertussisvakzine das Atopierisiko deutlich erhöhte (Odds Ratio = 1,76). Ein leicht erhöhtes Atopierisiko ergab sich auch für Kinder, die früh mit Antibiotika behandelt wurden, umgekehrt schien eine durchgemachte Maserninfektion vor einer Atopie zu schützen.

Doch warnen Pädiater davor, irgendeine Schlußfolgerung aus diesen Ergebnissen abzuleiten. Sie verweisen darauf, daß es sich um eine retrospektive Untersuchung handelt, bei der lediglich nach statistischen Zusammenhängen gesucht wurde. Die gefundenen Korrelationsdaten seien zwar interessant, doch sollte man diese Untersuchung sorgfältig einer kritischen Beurteilung unterziehen und die gefundene statistische Assoziation in weiteren Studien auf ihre klinische Bedeutung hin abklopfen. Auf jeden Fall sollten die Ergebnisse die Ärzte nicht davon abhalten, Kinder gegen potentiell lebensbedrohliche Erkrankungen zu impfen. 